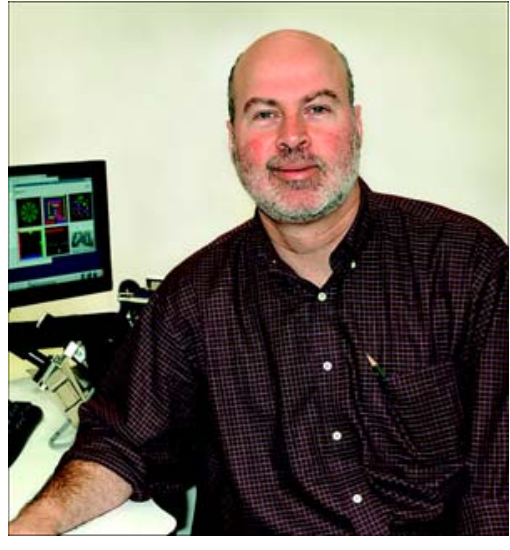

Hermano Igo Krebs, PhD

\title{
Those magnificent men and their flying machines
}

\section{INTRODUCTION}

I was inspired when I overheard, by chance, a conversation between the daughters of two subjects who had suffered strokes in the past year and were enrolled in my study at the Burke Rehabilitation Hospital (White Plains, New York). This study, performed in collaboration with my colleague Dr. Bruce Volpe, is sponsored by the National Institute of Child Health and Development/National Center for Medical Rehabilitation Research. While waiting for their loved ones to complete robotic sessions, one daughter confided to the other that her parents had had strokes 15 years apart and that she was quite impressed with the improved acute care her mother received. Then she reflected for a moment and, in a sad tone, observed that both parents' rehabilitation processes, however, were almost exactly the same. From her perspective, nothing had actually changed in that 15-year interval.

She is right. So far, no "magic bullet" exists for rehabilitation following stroke. The last 75 years of rehabilitation practice and research have delivered few actual answers for ameliorating and maximizing favorable outcomes for stroke survivors. We have essentially perpetuated long-time rehabilitation practices, many of which fall more under the realm of art than science.

However, looking into my "crystal ball” and moving the clock forward 14 years to 2020, I predict that the daughter's comments might be quite different then. Looking beyond the marble floors recently laid in every U.S. rehabilitation hospital, beyond the glass and glitter of the corridors and the amenities in patients' rooms, I am optimistic that we are at the cusp of a major transformation in physical medicine. New tools for novel neuroprotection agents, imaging techniques, robotics, electrical stimulation, neurostimulation, nerve growth factors, neurorecovery agents and, ultimately, neuron genesis and replacement will change the way we practice rehabilitation medicine and significantly raise our expectations from the present limited goal of disability management to an actual cure.

Rehabilitation robotics is one agent of change that will be ready for fullscale deployment within the next year or two. True, if one's expectation of robotics is inspired by "Commander Data" of the television series Star Trek: The Next Generation or by movies such as Terminator, then we are still falling short of our actual capabilities. A much better rendering of what I expect to see in rehabilitation hospitals and wellness facilities is a gymnasium (gym) of robots. This gym would not be very distinct from today's top-of-the-line gyms except that robots would assist people with disabilities as appropriate, possibly via real-time sensing or imaging techniques. The clinician would choose the therapy goals and approach and then supervise or teach the patient how to achieve those goals. The patient would bring robotic tools home, and these robotic platforms would become the common denominator in the continuum of care that includes the acute facility, rehabilitation hospital, outpatient clinic, 
wellness center, and home. Envision the use of robotics moving beyond care: social environments could be established via the Internet and patients could interact, compete against one other, and thrive.

\section{0? AM I AN OPTIMIST?}

Prior to 1990, no articles on robotic therapy had been published. Of course, the application of robotics to rehabilitation has a longer history, but the strong and sustained growth of activity in recent years is the result of a significant shift away from assistive technology for people with disabilities (conceptually, "smart" versions of a crutch) toward robotic therapy that supports and enhances clinicians' productivity and effectiveness in facilitating patient recovery. The magnitude of this change goes far beyond the usual ebb-and-flow of activity in technology-related fields. For example, the approximate number of articles submitted to the biennial International Conference on Rehabilitation Robotics (ICORR) from 1997 to 2005 demonstrates a sharp upswing in interest in rehabilitation robotics. At the latest ICORR (July 2005, Chicago, Illinois), approximately 80 percent of the accepted articles addressed rehabilitation robotics, up from approximately 33 percent at all the four preceding ICORRs.

This sharply increased activity is understandable: the demand for rehabilitation services is growing in pace with the "graying" of the population. By 2050, the contingent of U.S. seniors is expected to double from 40 to 80 million. With this growth comes increased incidence of age-related pathologies, including cerebral vascular accident (stroke). The numbers for Europe paint a similar picture. The Pacific Rim countries may face even greater challenges. For example, the percentage of the Japanese population aged 65 and older is projected to increase to 30 percent by 2025 and to 36 percent by 2050 . This situation creates an urgent need for new approaches that improve the effectiveness and efficiency of rehabilitation. It also creates an unprecedented opportunity for the deployment of technologies such as robotics for assisting in the recovery process.

\section{SO WHAT IS STATE OF THE ART IN REHABILITATION ROBOTICS?}

In this special $J R R D$ issue, I wanted to offer researchers three views of the Federal agencies that support most rehabilitation robotics research. Perception can be confused with reality, and I believe it is critical to prepare newcomers for hardship so they do not ride the emotional roller coaster that ranges from euphoria over the multitude of research opportunities to despair of finding resources to carry them out. I also want to emphasize that the realization of researchers' visions requires lifelong commitment to the rehabilitation field. In this way, the future will always be brighter than the past. This commitment to and appreciation of the need for continuing education and training of new generations was best exemplified by the late Robert W. Mann, who founded the Newman Laboratory for Biomechanics and Human Rehabilitation at the Massachusetts Institute of Technology (MIT) and to whom this issue is dedicated.

This issue continues with a brief technical/clinical overview of state-of-the-art rehabilitation robotics. I admit that it constitutes but a limited overview of the field. Just as in a "wine-tasting" workshop, though, the goal is to leave the reader with a taste for learning more about the topic. The limited number of examples cited here does not do justice to the field's "brave heart" researchers, but I tried to present a broad spectrum within two major categories: upper- and lower-limb robotics, which offer different shades of technological sophistication. The selection of research groups allows the reader to compare the present issue to a $2000 J R R D$ issue that my MIT colleague Professor Neville Hogan organized. The reader can readily appreciate how much the field has matured in the last 5 years.

The technical and clinical overview starts with two articles on assistive technology. Looking beyond the intended applications of Rahman et al.'s (p. 583) and Herder et al.'s (p. 591) devices, one can envision combining these devices with visual feedback. This combination would transform these lowcost devices into therapy tools for persons with milder symptoms. The next set of articles focuses on 
upper-limb robotics. Hogan et al. (p. 605) discuss intriguing results that support the view that the central nervous system cares less about muscles and more about motor learning. Kahn et al. (p. 619) compare robot-assisted versus unassisted training. Lum et al. (p. 631) compare provocative results on unilateral versus bilateral training, and finally, Patton et al. (p. 643) challenge us to think "outside the box" by using erroraugmentation, instead of error-free, approaches to promote recovery. The following three articles represent the most prolific research groups for lower-limb therapeutic robotics. Reinkensmeyer et al. (p. 657) discuss efforts to provide robotic tools that train animals and humans and allow experimentation and transference of the findings from the animal to human model. Riener et al. (p. 679) discuss new control schemes that significantly expand the interactive capabilities of the Lokomat gait trainer and make it more responsive to the patient, while Hesse et al. (p. 671) provide a remarkable tour de force on clinical results for gait training with the Gait Trainer I. The last article by Carignan et al. (p. 695) provides an exciting overview of this brave new world and goes beyond a "gym of robots" for the clinic.

As I mentioned earlier, the image of rehabilitation robotics as the eventual common link along the rehabilitation healthcare chain is not far-fetched. Indeed, I predict that in the near future patients will bring home therapy devices to which they were introduced in the hospital and will continue their care from home via the Internet. Patients with similar disabilities will form social networks to meet, play games, and compete with one other from the comfort of their homes.

If my oracle does not fail me, the researcher's, particularly the clinical researcher's, challenge for the next 5 or 10 years is to identify the variables that influence rehabilitation outcome, determine how they interact, and evaluate their impact on outcomes. If we make significant inroads into this facet, then I can rest assured in my prediction that a radical transformation in physical medicine practice is achievable.

Jump ahead to 2020 and just think what those "users" (patients, family members, caregivers, clinicians) at Burke and Spaulding Rehabilitation Hospitals and at the Baltimore and West Haven Department of Veterans Affairs Medical Centers will say when they compare notes.

\section{Hermano Igo Krebs, PhD}

Department of Mechanical Engineering, Massachusetts Institute of Technology, Cambridge, MA; Department of Neuroscience, The Winifred Masterson Burke Medical Research Institute, Joan and Sanford I. Weill Medical College of Cornell University, New York, NY

Email: hikrebs@mit.edu

DOI: 10.1682/JRRD.2006.08.0093 